\title{
Effects of discrete acoustic prestimuli on perceived intensity and behavioral responses to startling acoustic and tactile stimuli
}

\author{
NEAL R. SWERDLOW and MARK A. GEYER \\ University of California, San Diego, La Jolla, Califormia \\ TERRY D. BLUMENTHAL \\ Wake Forest University, Winston-Salem, North Carolina \\ and \\ PATRICIA L. HARTMAN \\ University of California, San Diego, La Jolla, California
}

\begin{abstract}
Startle magnitude is reduced when a startling stimulus is preceded $30-500 \mathrm{msec}$ by a weak prepulse. Although the neurobiology of prepulse inhibition (PPI) of the startle reflex has been studied across species, relatively little is known about the relationship between reduced PPI and informationprocessing abnormalities in specific neuropsychiatric disorders. In one conceptual model of PPI, the reduced responsivity to sensory events after a prepulse protects the information contained in the prepulse, so that it can be processed adequately. Thus deficient PPI might reflect reduced informationprotective abilities and contribute to cognitive dysfunction. Nevertheless, there is little concrete evidence to support this conceptual model. In the present experiment, the relationship of PPI and perceived stimulus intensity was investigated, using both unimodal and cross-modal stimuli to elicit startle and PPI in nonpsychiatric human subjects. The subjects rated the intensity of a startle stimulus presented alone or $60 \mathrm{msec}$ after the onset of an acoustic prepulse versus the intensity of an identical startle stimulus alone, presented $5 \mathrm{sec}$ earlier or later. A negative relationship was observed between the amount of PPI produced by a range of weak-to-intense acoustic prepulses and the perceived intensity of either acoustic or tactile startle stimuli. These findings support an information-protective model for the impact of weak prestimuli on perceived startle stimulus intensity and suggest that deficient PPI in neuropsychiatric disorders may be accompanied by a reduced ability to automatically inhibit, or gate, sensory information.
\end{abstract}

Prepulse inhibition (PPI) is the reduction in startle magnitude that occurs when a startling stimulus is preceded $30-500 \mathrm{msec}$ by a weak prestimulus, or prepulse. PPI has been used as an operational measure of sensorimotor gating: The degree to which a response to an intense stimulus is inhibited by the weak sensory event (prepulse) is the measure of the amount of sensorimotor gating. Thus, the relative loss of PPI in specific neuropsychiatric disorders, including schizophrenia (Braff, Grillon, \& Geyer, 1992; Braff et al., 1978), Huntington's disease (Swerdlow, Paulsen, et al., 1995), and Tourette syndrome (Castellanos et al., 1996), has been interpreted as evidence for deficiencies of sensorimotor gating in these disorders.

This study was supported by NIMH Grants MH 01436, MH 54621 , MH 42228 and an Independent Investigator Award to N.R.S. from the National Alliance for Research on Schizophrenia and Depression (NARSAD). The authors gratefully acknowledge the assistance of Pamela Auerbach and Navid Taaid in the completion of these studies and the preparation of this manuscript. Correspondence concerning this article should be addressed to N. R. Swerdlow, Department of Psychiatry, University of California, San Diego, School of Medicine, 9500 Gilman Drive, La Jolla, CA 92093-0804 (e-mail: nswerdlow@ucsd.edu).
In one conceptual model of PPI, a weak stimulus activates brain-based processes that initially increase and then blunt responsivity to sensory events during a subsequent brief temporal window; the period of gating is empirically determined to be approximately $30-500 \mathrm{msec}$ in duration, in both rats and humans (Graham, 1975; Ison, McAdam, \& Hammond, 1973). This period of reduced responsivity might momentarily protect the information contained in the weak stimulus, so that it can be processed adequately, without interference from subsequent stimuli (Norris \& Blumenthal, 1996; Perlstein, Fiorito, Simons, \& Graham, 1993). Some experimental evidence supports this information-protective hypothesis: Norris and Blumenthal reported that subjects most accurately identified the presence of a prepulse stimulus on trials with maximal PPI. In other words, reflex inhibition was accompanied by a decreased ability of a startling pulse to interrupt preattentive processing of the prepulse (Norris \& Blumenthal, 1996).

Although this information-protective model has been proposed to explain the relationship between PPI deficits and cognitive impairment in neuropsychiatric disorders, the relationship between PPI and the perceived intensity of sen- 
sory information (stimuli) is quite complex. Theoretically, some relationship between sensorimotor gating and sensory gating might be expected by the overlap in brain substrates believed to regulate these two processes. Specifically, the hippocampus has been implicated in the regulation of both PPI (Caine, Geyer, \& Swerdlow, 1992; Swerdlow, Lipska, et al., 1995) and P50 inhibition (Bickford-Wimer et al., 1990), the latter of which has been interpreted as a measure of sensory gating. Furthermore, both PPI and P50 gating are disrupted in certain clinical conditionssuch as schizophrenia (Braff et al., 1992; Braff et al., 1978; Siegel, Waldo, Mizner, Adler, \& Freedman, 1984)in which hippocampal pathology is implicated (Conrad, Abebe, Austin, Forsythe, \& Scheibel, 1991). More direct experimental evidence also links prestimulus-induced gating of sensory and motor information. Cohen, Hoffman, and Stitt (1981) reported that an acoustic prepulse reduced the startle response elicited by a forehead tap, as well as the perceived intensity of that tap. Perlstein et al. (1993) reported a modest reduction in the perceived intensity of a 110-dB acoustic startle stimulus when it was preceded $120 \mathrm{msec}$ earlier by a $75-\mathrm{dB}$ acoustic prepulse. The extent of the reduction in the perceived intensity - approximately $12 \%$, on average - was small but statistically significant.

Other findings, however, raise questions about the generalizability of the information-protective model of PPI. Blumenthal and colleagues (Blumenthal, Schicatano, Chapman, Norris, \& Ergenzinger, 1996) reported that either the perceived intensity of acoustic startle stimuli is unchanged or reductions in perceived intensity are evident only when the startle stimulus and the prepulse are in the same stimulus modality (in contrast to the findings of Cohen et al., 1981 ) and differ by at least $30 \mathrm{~dB}$ in intensity (in agreement with the findings of Perlstein et al., 1993). Their findings support the conclusion that the reported withinmodality reduction in perceived stimulus intensity was likely to be a result of loudness assimilation, a form of stimulus intensity averaging that caused the perceived intensity of the stimulus to reflect the assimilated, or averaged, intensity of the weak prestimulus and the more intense startle stimulus. As described by Perlstein et al., loudness assimilation happens when the "loudness of pair members shifted toward one another" (p. 356). Furthermore, Blumenthal et al. reported that "when the prepulse and startle stimulus are in different sensory modalities, the two are independent" (p. 80). Although these conclusions leave room for the possibility that, under certain circumstances, PPI may be the result of information-protective brain processes, such circumstances would appear to be limited to intramodal (within-modality) acoustic information, in which prepulse and pulse differed substantially in intensity.

Because of the potential importance of the concept of information-protective sensorimotor gating in the interpretation of PPI deficits in neuropsychiatric populations, we undertook a series of studies to investigate the relationship of PPI to perceived stimulus intensity, using both intramodal (acoustic prepulse and acoustic pulse) and cross-modal (acoustic prepulse and tactile airpuff) stimuli to elicit startle and PPI in nonpsychiatric human subjects. Subjects rated the intensity of a startle stimulus presented alone or $60 \mathrm{msec}$ after an acoustic prepulse versus the intensity of an identical startle stimulus alone, presented $5 \mathrm{sec}$ earlier or later. Both subjective intensity ratings and bilateral electromyographic measures of orbicularis oculi (eyeblink) activity were recorded from each subject. We report a strong relationship between the amount of PPI elicited by a range of weak-to-intense acoustic prepulses and the perceived intensity of either acoustic $(118 \mathrm{~dB})$ or tactile (40 psi) startle stimuli. These findings are interpreted in relation to both information-protective and loudness assimilation theories for the impact of weak prestimuli on perceived startle stimulus intensity.

\section{METHOD}

\section{Subjects}

Subjects $(N=41)$ were recruited via advertisement within the San Diego community (ratio of males to females, 29:12; mean age, 29.07 years; range, 18-50 years). Hearing was screened in accordance with our usual protocol (Braff et al., 1992; Saico Audiometer [Assens, Denmark]; exclusion: impairment at $40 \mathrm{~dB}(\mathrm{~A}), 1 \mathrm{kHz}$ ). Urine toxicology was completed on some subjects $(n=14)$; no illicit substances were detected in these individuals. Because comparisons between subgroups with and without toxicological screens revealed no differences, the subgroups were combined for all analyses.

\section{Procedure}

Testing was performed during the light phase $(0900-1700$; room temperature, $68^{\circ}-70^{\circ} \mathrm{F}$ ), using an SR-LAB PC computer monitoring system and custom EMG amplifier with a $1 \mathrm{kHz}$ band-pass filter (SDI, San Diego, CA). The subjects sat in a quiet room, and eyeblink EMG was measured bilaterally. Two Beckman miniature Ag$\mathrm{AgCl}$ electrodes $(\mathrm{R}<15 \mathrm{~K} \Omega$ ) were placed below each eye, over the orbicularis oculi, $1 \mathrm{~cm}$ inferior to the external canthus on each side. A ground electrode was placed behind the left ear, over the mastoid. The subjects wore Maico headphones (Minneapolis, MN) and looked at a point on the wall, approximately $1 \mathrm{~m}$ from the floor, that allowed them to be comfortable with their eyes open. On the wall $1 \mathrm{~m}$ from their eyes was a linear scale, $64 \mathrm{~cm}$ in length, with numbers ( $1.5 \mathrm{~cm}$ height) and 1.0-unit gradations dividing the line from a minimum value of -10 units to a maximum value of +10 units. The words much less, less, and slightly less were written under the scale locations corresponding to $-10,-5$, and -1.5 , respectively; the words much more, more, and slightly more were written under the scale locations corresponding to 10,5 , and 1.5 , respectively. The subjects participated in either acoustic startle testing or tactile startle testing; no subject was tested in both paradigms. The paradigms were identical in every way, except for the nature of the startling stimuli (acoustic vs. tactile).

The subjects were informed that they would hear pairs of noise bursts (in the Noise group; $n=20$ ) or feel pairs of air puffs to the sternal notch (in the Puff group; $n=21$ ), separated by $5 \mathrm{sec}$. The subjects were oriented to the linear scale mounted in front of them and were told that, for each pair of stimuli, they should mentally assign the first stimulus an intensity value of 0 and should rate the intensity of the second stimulus relative to the first stimulus in the pair. If the two stimuli appeared to have equal intensity, the proper rating assignment would be 0 . If the second stimulus appeared more intense than the first stimulus, the proper rating assignment would be 
a positive number, up to +10 , depending on the degree of perceived intensity difference. If the second stimulus appeared less intense than the first stimulus, the proper rating assignment would be a negative number, down to -10 , depending on the degree of perceived intensity difference. Ratings were made as verbal responses after each trial pair and were recorded by an experimenter (P.L.H.), who was located in the same room, seated behind a sound-attenuation barrier, out of sight of the test subject.

For all testing, a 5-min 70-dB(A) background white noise acclimation period was followed by acoustic or air puff startle stimuli. Acoustic stimuli included (1) a 118-dB(A) 40-msec noise burst (Noise) or (2) 20-msec prepulse noises that were $4,8,12$, or $16 \mathrm{~dB}(\mathrm{~A})$ above background (PP4, PP8, PP12, or PP16). Air puffs were 40-psi, 40-msec air bursts (Puff) aimed at the sternal notch via PE tubing attached to a customized frame. Noise bursts or air puffs were presented either alone or $60 \mathrm{msec}$ after the onset of the prepulse.

The stimuli were presented in pairs, with each pair being termed a trial. In some cases, pairs consisted of two identical startle stimuli (eg. either Noise followed by Noise or Puff followed by Puff). Ratings on these trials served as reference ratings for the calculation of changes in perceived stimulus intensity. For other pairs, one stimulus was always a startle stimulus alone, and the other stimulus was a startle stimulus preceded by a $4-16 \mathrm{~dB}(\mathrm{~A})$ prepulse. Trials that included a prepulse-containing stimulus were of two types: those in which the prepulse-containing stimulus was the second of the trial (Order A), and those in which the prepulse-containing stimulus was the first of the trial (Order B; Figure 1). Sessions thus included a total of nine trial types: one reference trial and trials of Orders $A$ and B for 4-, 8-, 12-, and 16-dB prepulses. Stimuli were presented in a pseudorandom order, with an equal number of each prepulse intensity presented in the first and second stimulus of the pairs. Sessions included a total of 54 trials (i.e., 108 startle stimuli), with an average intertrial interval of $20 \mathrm{sec}$ (range, 16-24 sec).

The impact of the prepulse on estimates of startle stimulus intensity was calculated as the numerical difference between the rating assigned under conditions in which both stimuli were startle stimuli (reference trials) and the ratings assigned under conditions in which one stimulus included a prepulse (Figure 1). Because the subjects were always asked to rate the intensity of the second stimulus in the trial, relative to the first stimulus in the trial, prepulse rating inhibition (PPRI) was reflected by relatively lower ratings in the situation in which the prepulse-containing stimulus was the second of the trial (Order $\mathrm{A}$ ) and by relatively higher ratings when the prepulsecontaining stimulus was the first of the trial pair (Order B). For Order $\mathrm{A}, \mathrm{PPRI}_{\mathrm{A}}=[$ Reference $]-[\operatorname{Order} \mathrm{A}]$; for Order $\mathrm{B}, \mathrm{PPRI}_{\mathrm{B}}=$ [Order B] $-[$ Reference $]$; and overall, PPRI $=\left[\mathrm{PPRI}_{\mathrm{A}}+\mathrm{PPRI}_{\mathrm{B}}\right] / 2$. In this way, larger PPRI values signify a relatively stronger impact of the prepulse on reducing the perceived intensity of the startling stimulus. For some calculations, PPRI values were normalized by accounting for the total range of ratings provided by each subject. For these calculations, normalized PPRI $(\mathrm{nPPRI})=[\mathrm{PPRI} / \mathrm{rating}$ range] $\times 100$.

Digitized blink responses were displayed on the PC monitor; voluntary and spontaneous blinks were excluded on the basis of published criteria (Braff et al., 1992). Reflex characteristics included mean startle magnitude, onset and peak latency, \% acoustic PPI $[100-100$ (magnitude on PP + Noise trials)/(magnitude on Noise trials)], and \% tactile PPI [100 - 100(magnitude on PP + Puff tri-
S1
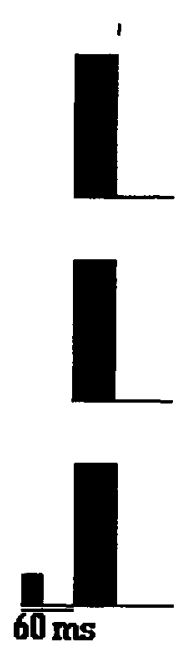

S2

5 seconds

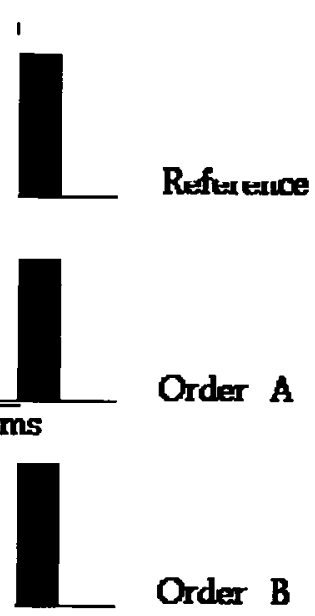

Figure 1. Schematic representation of stimulus configurations for acoustic and tactile startle sessions. Stimuli were delivered in pairs, S1 and S2, separated by $5 \mathrm{sec}$. The subjects were told to rate the intensity of the $S 2$, relative to $S 1$, on a scale of -10 (much weaker) to +10 (much more intense). Prepulse rating inhibition (PPRI) was calculated as a difference score of the ratings as follows: Order A: PPRI $_{A}=\left[\right.$ Reference] minus [Order A]; Order B: PPRI $I_{B}=[$ Order B] minus [Reference]; Overall PPRI $=\left[P P R I_{A}+P_{P R I}\right] / 2$. In this way, larger PPRI values signify a relatively stronger impact of the prepulse on reducing the perceived intensity of the startling stimulus. For some calculations, PPRI values were normalized by accounting for the total range of ratings provided by each subject. For these calculations, normalized PPRI (nPPRI) $=[$ PPRI/rating range $] \times 100$. Thus, this nPPRI value reflected the percent reduction in perceived intensity of a stimulus, relative to the individual's idiosyncratic range of perceived intensities. 
als)/(magnitude on Puff trials)]. PPI scores for both acoustic and tactile startle sessions were analyzed by mixed design analysis of variance (ANOVAs), with stimulus order and prepulse intensity as within-subjects measures. As in previous reports (Braff et al., 1992; Swerdlow, Filion, Geyer, \& Braff, 1995; Swerdlow, Hartman, \& Auerbach, 1997; Swerdlow, Paulsen, et al., 1995), subjects whose mean startle magnitude was $<10(1$ unit $=0.77 \mu \mathrm{V})$ for Block 1 or 2 were identified as nonresponders, and their data were not analyzed further. Most nonresponders had low or zero startle magnitudes in Block 2; informal inspection of their data in Block 1 revealed patterns of both PPI and PPRI consistent with the two-block data from startle responders. Final group sizes for acoustic and tactile startle measures (both blocks) were each $n=14$. The loss of subjects due to nonresponder criteria $(31.7 \%)$ is greater than the typical rates (approximately $15 \%$ ) in our previous reports, perhaps owing to the particular stimulus configurations in this study, the greater number of stimulus trials ( 108 vs. approximately 50 ) and, hence, the greater reflex habituation, and the use of bilateral eyeblink measures (in some cases, startle magnitude criteria were met for one, but not both eyes).

Owing to the subjective scaling of rating measures, several different strategies were used to explore the effects of prepulses on intensity ratings. All of these different strategies yielded comparable findings but are included to demonstrate the stability of the out- comes across different statistical approaches. PPRI was first analyzed via nonparametric statistics. To determine whether ratings varied significantly from 0 (i.e., Stimulus A and Stimulus B rated as being of equal or different intensity), a one-sample sign test was applied to each set of stimulus pair conditions. PPRI was then compared among prepulse intensities, using a Wilcoxon signed-rank test. To examine correlations between PPI and PPRI (and nPPRI) across subjects, the mean amount of PPI and PPRI (and nPPRI) exhibited by each subject (collapsed across all the prepulse conditions) was ranked and submitted to a Spearman rank correlation analysis. Values for PPRI and nPPRI were also submitted to parametric analyses, using an ANOVA, with repeated measures on prepulse intensity $(4,8,12$, or $16 \mathrm{~dB}$ above background). PPRI and nPPRI were also ranked across the four prepulse intensities for each subject, and these ranking data were submitted to an ANOVA, with repeated measures on prepulse intensity. For all comparisons, alpha was .05 .

\section{RESULTS}

Startle magnitudes for acoustic (Noise) and tactile (Puff) reference trials are seen in Figure 2. Startle magnitude was comparable whether the startle stimulus was pre-

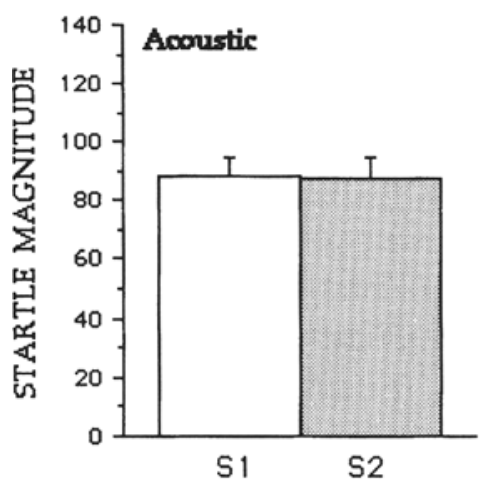

\section{INTENSITY RATING \\ ( $\mathrm{S} 2$ relative to $\mathrm{S} 1$ )}
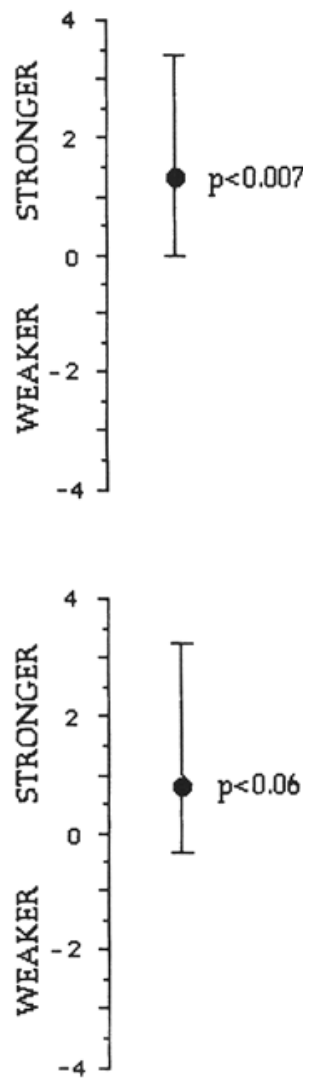

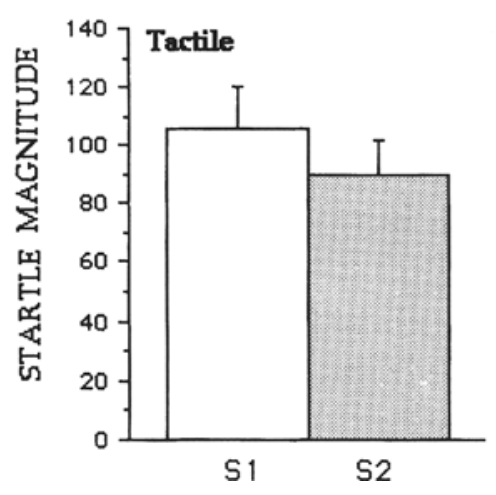

Figure 2. Dissociation of startle magnitude from perceived stimulus intensity in reference trials. Startle magnitude (mean $+S E M$ ) and stimulus intensity estimates (median \pm quartile ranges) for stimulus pairs in which $S 1$ and $\mathbf{S 2}$ are both either Noise (acoustic) or Puff (tactile) are given. Whereas startle magnitude either remained unchanged or diminished slightly between $\mathbf{S 1}$ and $\mathbf{S 2}$, intensity estimates for $\mathbf{S 2}$ reflected an increase in the perceived magnitude of $S 2$, relative to $S 1$. 
sented as the first or the second stimulus of a trial pair: when stimulus pairs consisted only of two Noise or two Puff stimuli, an ANOVA revealed no significant effect of order for either acoustic startle $(F<1)$ or tactile startle $[F(1,13)=2.53$, n.s.]. An ANOVA of these Noise pairs or these Puff pairs also revealed no significant effect of eye (right vs. left: $F<1$, both modalities), a significant effect of block [acoustic: $F(1,13)=24.51, p<.0005$; tactile: $F(1,13)=35.73, p<.0001]$, and no significant order $\times$ block or order $\times$ eye interaction ( $p>.05$, all comparisons, both modalities).

An ANOVA of acoustic startle magnitude under all trial conditions (Noise stimuli included in reference trials, as well as those included in mixed trials) revealed a significant effect of block and stimulus order but neither significant effects of eye nor any significant two- or three-way interactions (all the significant effects are shown in Table 1). Habituation led to a significant reduction in the magnitude across blocks for both acoustic startle [mean values for Block 1 vs. Block $2=99.97$ vs. 68.51 (SEMs = 5.92 and 5.22)] and tactile startle [mean values for Block 1 vs. Block $2=123.26$ vs. $42.82(S E M s=10.15$ and 4.86$)]$; however, in neither case did startle values approach floor levels in Block 2. A similar analysis of tactile startle magnitude also revealed significant effects of block and stimulus order but no significant effects of eye and no significant two- or three-way interactions (Table 1). For both acoustic and tactile startle measures, the significant effects of block reflected reflex habituation across the startle session, and the significant effect of order reflected lower Noise or Puff intensity ratings for Order B (i.e., when the stimulus was the second of the pair), as compared with Order A.

Values for the rating ranges for acoustic (Noise stimuli) and tactile (Puff stimuli) startle sessions are seen in Table 2 . A distinction was observed between the order effects on the startle reflex magnitude and those on stimulus intensity ratings. Despite the lack of order effect on startle response magnitude to paired Noise or Puff stimuli, the subjects tended to rate the intensity of the second stimulus to be greater than that of the first stimulus. In pairs of Noise trials, the median rating of the second stimulus was +1.33 ( $p<.007$, one-sample sign test); for Puff pairs, the median rating of the second stimulus was +0.78 , which only approached statistical significance $(p<.06$, onesample sign test; Figure 2). The statistical outcomes were identical when these ratings were normalized for individual rating ranges.

Prestimuli caused graded inhibition of both acoustic and tactile startle, which was independent of stimulus order (i.e., whether prepulse + Noise (or prepulse + Puff) stimulus was first or second in each trial). An ANOVA of PPI revealed significant effects of prepulse intensity (acoustic, $F=62.14$; tactile, $F=23.93$ ) but no significant effect of order (acoustic, $F=1.40$; tactile, $F<1$ ), eye (acoustic, $F<1$; tactile, $F=2.21)$, or trial block $(F<1$, both modalities). Additional significant interaction effects are noted in Table 1. The lack of a significant trial block effect on PPI [mean acoustic PPI (for) Block 1 vs. Block $2=$ 42.83 vs. $38.48(S E M s=2.18$ and 2.93$)$; mean tactile PPI (for) Block 1 vs. Block $2=58.48$ vs. 61.11 (SEMs $=2.58$ and 4.09)] may reflect the fact that the present PPI calculations are based on a percent reduction in startle magnitude on prepulse + Noise trials, relative to startle magnitude on Noise trials; when PPI is instead calculated as the arithmetic difference between startle magnitude on Noise and prepulse + Noise trials, PPI has been shown to diminish as startle magnitude habituates (Blumenthal, 1997). Inspection of the data (Figure 3, top) reveals a monotonic relationship between prepulse intensity and the amount of PPI of acoustic startle and an inverted-U relationship between prepulse intensity and PPI of tactile startle.

The impact of prepulses on intensity ratings of the acoustic and tactile stimuli (PPRI) are seen in Figure 3 (bottom). The impact of prepulses on perceived stimulus intensity was weak. Median PPRI values for the optimal prepulse intensities were 0.92 for acoustic startle and 0.67 for tactile stimuli, both of which correspond to slight re-

Table 1

Significant Main and Interaction Effects in an Analysis of Variance of Acoustic and Tactile Startle

\begin{tabular}{cllrcc}
$\begin{array}{c}\text { Measure } \\
\text { Startle amplitude }\end{array}$ & Modality & & \multicolumn{1}{c}{ Effect } & $d f$ & $p$ \\
& Acoustic & Block & 40.63 & 1,13 & $<.0001$ \\
& & Order & 8.86 & 1,13 & $<.015$ \\
& \multirow{2}{*}{ Tactile } & Block & 30.28 & 1,13 & $<.0001$ \\
& & Order & 31.08 & 1,13 & $<.001$ \\
Prepulse inhibition & Acoustic & Intensity & 62.14 & 3,39 & $<.0001$ \\
& & Block $\times$ order & 5.30 & 1,13 & $<.05$ \\
& \multirow{4}{*}{ Tactile } & Eye $\times$ order $\times$ intensity & 3.01 & 3,39 & $<.05$ \\
& & Intensity & 23.93 & 3,39 & $<.0001$ \\
& & Block $\times$ orde। & 5.52 & 1,13 & $<.05$ \\
& & Eye $\times$ order $\times$ intensity & 3.67 & 3,39 & $<.05$ \\
& & Block $\times$ order $\times$ intensity & 5.94 & 3,39 & $<.002$ \\
& & Eye $\times$ block $\times$ order $\times$ intensity & 6.09 & 3,39 & $<.002$
\end{tabular}


Table 2

Intensity Ratings (Means and Ranges) for Acoustic (Noise) and Tactile (Puff) Stimuli

\begin{tabular}{lccccccc} 
& \multicolumn{2}{c}{ Minimum } & \multicolumn{2}{c}{ Maximum } & \multicolumn{2}{c}{$\begin{array}{c}\text { Rating Range } \\
\text { (Max }- \text { Min) }\end{array}$} \\
Modality & $M$ & Range & $M$ & Range & $M$ & Range \\
Acoustic & -2.93 & $(-5)-(-1)$ & 5.29 & $3-8$ & 8.21 & $5-13$ \\
Tactile & -4.14 & $(-9)-0$ & 5.43 & $1-10$ & 9.57 & $3-15$
\end{tabular}

ductions in perceived intensity, according to the visual analogue scale utilized in testing; median nPPRI values under these conditions were 0.105 and 0.096 , indicating median values of $10.5 \%$ and $9.6 \%$ reductions in perceived intensity of acoustic and tactile stimuli, respectively.

Despite the weakness of this effect, there was an orderly monotonic relationship between prepulse intensity and the amount of PPRI for acoustic stimuli and an inverted-U relationship between prepulse intensity and the amount of
PPRI for tactile stimuli. These patterns (and their statistical characteristics, see below) were evident, independent of whether PPRI data from startle nonresponders were included or excluded from the analysis. For acoustic stimuli, one-sample sign tests revealed significant PPRI for $16-\mathrm{dB}$ prepulses $(p<.015)$. Wilcoxon tests revealed that, as compared with $4-\mathrm{dB}$ prepulses, significantly more PPRI was elicited by $12-\mathrm{dB}(p<.025)$ and $16-\mathrm{dB}$ prepulses $(p<$ $.01)$. For tactile stimuli, one-sample sign tests revealed significant PPRI for $8-\mathrm{dB}$ prepulses $(p<.002)$. Wilcoxon tests revealed that $8-\mathrm{dB}$ prepulses elicited significantly more PPRI than did prepulses of either 4-dB $(p<.005)$ or 16-dB intensity $(p<.05)$. An ANOVA of PPRI for both acoustic and tactile stimuli revealed no significant effects of block or order or any interactions with prepulse intensity; thus, data were collapsed across these variables, revealing significant effects of prepulse intensity for acoustic $[F(3,37)=4.61, p<.008]$ and tactile stimuli $[F(3,39)=$ $2.85, p<.05]$. An ANOVA of nPPRI also revealed signif-

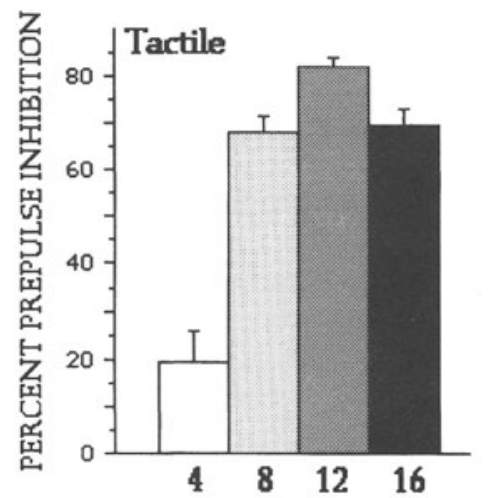

PREPULSE INTENSITY (dB(A))
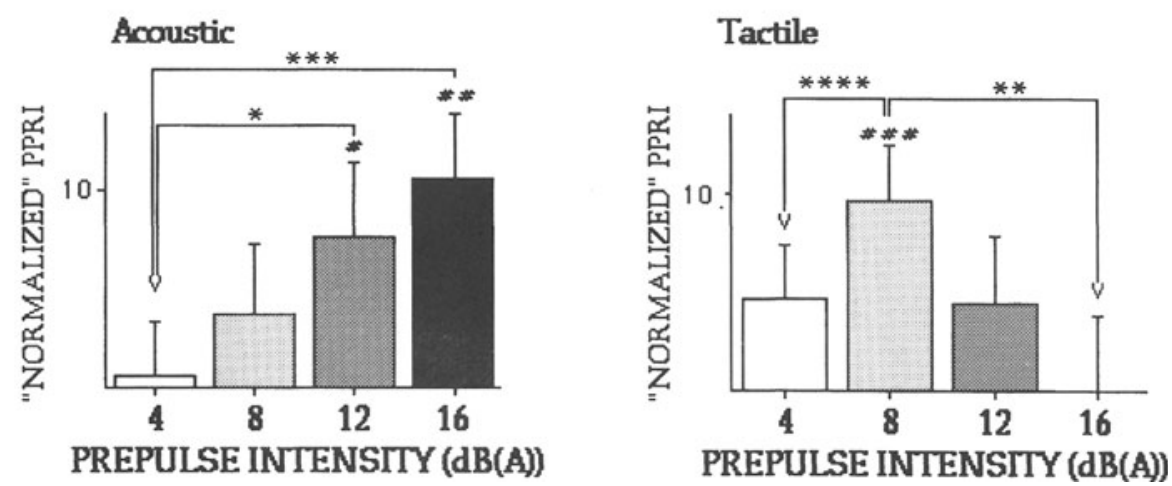

Figure 3. Correspondence of prepulse effects on startle magnitude and perceived stimulus intensity. Prepulse inhibition (PPI) and normalized prepulse rating inhibition (nPPRI) are given for acoustic and tactile startle. PPI and nPPRI values represent mean $+S E M .{ }^{*} p<.05 ;{ }^{* *} p<.01$; ${ }^{* \star *} p<.005 ; * \star \star \star p<.001$, one-factor ANOVA after significant main effect of intensity by repeated measures ANOVA. \#p<.025; \#p<.01; \#\# $<.002$, one-sample sign test. Other statistical approaches (parametric and nonparametric) to PPRI data yielded comparable results (see the text). 
icant effects of prepulse intensity for both acoustic $[F(3,39)=$ $6.02, p<.002]$ and tactile $[F(3,39)=4.71, p<.007]$ modalities. For both PPRI and nPPRI, post hoc comparisons verified patterns detected via nonparametric analyses of PPRI ( $p$ values, .01-.001; Figure 3). Finally, an ANOVA of ranked PPRI values verified significant effects of prepulse intensity for acoustic $[F(3,39)=6.29, p<.002]$ and tactile $[F(3,39)=3.21, p<.035]$ modalities, with post hoc comparisons being supportive of the nonparametric statistical findings.

Spearman analyses revealed significant correlations between the relative amounts of PPI and PPRI exhibited by subjects for tactile stimuli $(R=.58, p<.04)$, and a nearsignificant correlation for acoustic stimuli $(R=.51, p<$ $.07)$. One subject tested in the acoustic startle session reported no PPRI for any stimulus configuration (average PPRI $=-0.56$ units); without this subject, the correlation between acoustic PPI and PPRI was highly significant $(R=.75, p<.01$; Figure 4$)$. When each stimulus configuration was examined separately and the right and left eyes were examined separately, positive correlations between PPI and PPRI were noted for 15 out of the possible 16 trial configurations [mean $R=.36(S E M=.06)$ ]; the single (nonsignificant) negative correlation was for the lowest prepulse intensity ( $4 \mathrm{~dB}$, left eye, acoustic modality).

\section{DISCUSSION}

The present findings reveal both similarities and differences in the motor response to and the perceived intensity of a startling stimulus. Confirming the previous report of Perlstein et al. (1993), subjects consistently rated the second stimulus of any trial as more intense than the first stimulus of that pair, even though startle response magnitude on those trials was not affected by stimulus order. Conceivably, higher intensity ratings for the second stimulus in the pair may result from a form of facilitatory priming, in which the first stimulus directs attention toward the second. When identical stimuli, presented in pairs, are judged to be of different intensity, this has been described as a time error (Peak 1939); when the second stimulus in the pair is judged to be more intense than the first, this is called a negative time error. Consistent with the present findings, Postman (1946) reported data from 4 subjects, in which identical paired acoustic stimuli separated by $4-6 \mathrm{sec}$ produced a negative time error-that is, the second stimulus was perceived to be more intense than the first. Negative time errors have been attributed to an additive effect of the perceived intensity of the second stimulus and a central aftereffect, or trace, of the first stimulus (cf. Postman, 1946; Woodworth \& Schlosberg, 1954).

Although the second stimulus in the present experiments was rated as more intense than the first stimulus when the two stimuli were identical in actual intensity (e.g., two Noise or two Puff stimuli), this difference was diminished when the second stimulus was preceded by a prepulse. Prepulses causing maximal PPI of the second star-

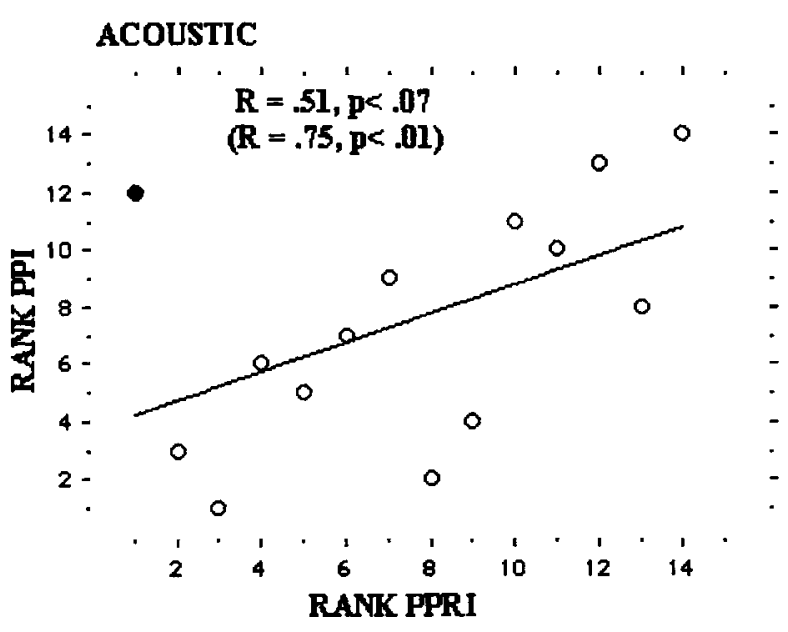

TACTILE

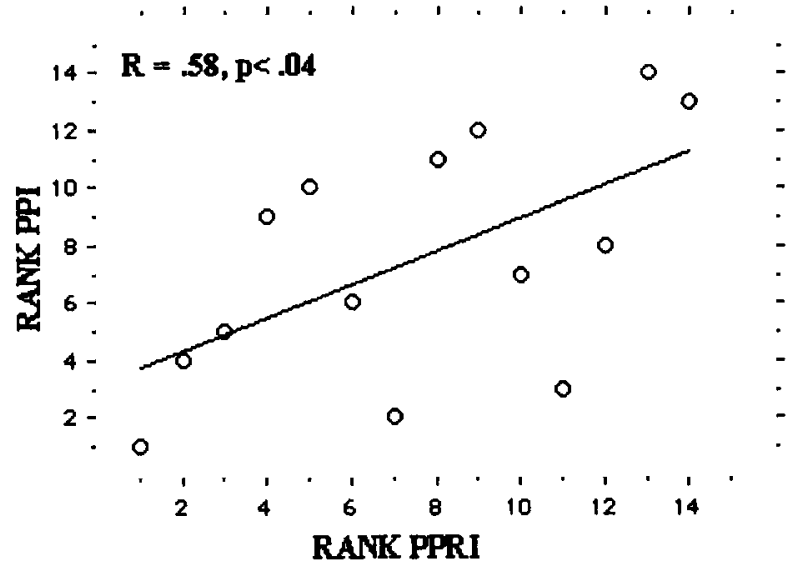

Figure 4. Distributions for Spearman rank correlation between prepulse inhibition (PPI) and prepulse rating inhibition are given (PPRI) for acoustic and tactile startle sessions. Correlations were statistically significant for tactile startle and approached significance for acoustic startle, reaching significance $(p<.01)$ only after elimination of single subject $(\bullet)$, who exhibited no PPRI under any stimulus conditions. Owing to the lack of clinical assessment of these test subjects, there is no useful information regarding the clinical characteristics of this outlier that would warrant statistical consideration.

tle response (i.e., 16-dB prepulses for acoustic startle and $12-\mathrm{dB}$ prepulses for tactile startle) resulted in perceived intensity ratings that approached 0 values - that is, the second stimulus was rated as being approximately equal in intensity to the first stimulus of the pair (in this case a Noise or a Puff trial).

On average, the second stimulus was never perceived as quieter than the first; yet prepulse-induced rating inhibition (PPRI) was evident in two ways: (1) if the second stimulus included a prepulse, it sounded less loud than the first stimulus than it would have had it not included a prepulse, and (2) if the first stimulus included a prepulse, the second stimulus sounded much louder than the first. The 
amount of PPRI was small, with magnitudes comparable with those reported previously by Perlstein et al. (1993), but it did correspond, at least modestly, with the motorreflex-inhibiting effects of the prepulse, for both acoustic and tactile startle. The correspondence between the amounts of PPRI and PPI was evident in the graded impact of prepulses on both of these variables and in the correlations in rank order of PPRI and PPI within subjects.

Prepulse intensity effects clearly differed across stimulus modalities, for both PPI and PPRI. The most intense prepulses $(16 \mathrm{~dB})$ maximally inhibited both the startle and the perceptual effects of acoustic stimuli, although these effects of tactile stimuli were maximally inhibited by weaker prepulses, 8 or $12 \mathrm{~dB}$ above background. In fact, the most intense acoustic prepulses appeared to produce no PPRI of tactile stimuli. One explanation for this observation is that, during tactile startle testing, acoustic prepulses have a greater salience as discrete events, because they are not followed by an intense noise burst. The most intense prepulses might, thus, be adequately salient to contribute to the perceived intensity of the startling experience, which primarily is being produced by the air puff. The ability of weaker acoustic prepulses to produce significant crossmodal PPRI suggests that a reduction in perceived intensity can occur by a mechanism other than simple loudness assimilation; the loss of this ability at higher prepulse intensities suggests that competing processes may increase as the acoustic prepulse becomes increasingly more salient. One test of this hypothesis would be to assess unimodal (acoustic) PPRI with more intense acoustic prepulses; this hypothesis would predict that, with adequately intense acoustic prepulses, an inverted-U-shaped intensity function would emerge.

Some evidence for a nonmonotonic impact of prestimulus intensity on perceived loudness has been reported in a paradigm utilizing unimodal acoustic stimuli. Using 5 subjects, 50 -msec pure acoustic tones, and prepulse intervals of 177 and $285 \mathrm{msec}$, Peak (1939) reported a monotonic relationship between prepulse intensity and reflex inhibition and an inverted-U-shaped relationship between prepulse intensity and the absolute impression of the loudness of the startling acoustic stimulus. The maximum amount of PPRI in that study, achieved at the shorter (177msec) prepulse interval, was approximately $23.7 \%$ somewhat larger than the maximum amount of PPRI obtained in the present studies. It is, thus, possible that a nonmonotonic relationship between prepulse intensity and PPRI in the present unimodal acoustic startle study might have emerged with different prepulse parameters. For example, maximal levels of unimodal PPI (approximately $70 \%$ ) and PPRI (approximately $10 \%$ ) in the present study, achieved with 16-dB prepulses, were approximately equal to the levels of cross-modal PPI and PPRI achieved with 8-dB prepulses. Prepulses that yielded greater cross-modal PPI (approximately $80 \%$ PPI with $12 \mathrm{~dB}$ ) produced PPRI that was beyond the peak of the inverted-U-shaped curve. Conceivably, acoustic prepulses capable of producing $80 \%$ unimodal PPI might have also exceeded peak values of PPRI, yielding an inverted-Ushaped function, similar to that observed in the present study with cross-modal PPI and in the study by Peak with unimodal PPI.

The ability of prepulses to blunt the perceived intensity of uni- and cross-modal startle stimuli may be consistent with an information-protective model of PPI. An alternative explanation for these findings is that the subjective perception of the intensity of a startle stimulus is strongly influenced by the magnitude of the startle reflex elicited by that stimulus. Subjects who experience a robust startle response (i.e., minimal or no PPI) might report the startling stimulus as more intense than a stimulus that produced a less robust startle reflex. In such a case, the strong correlation between PPI and PPRI would not reflect gating of a sensory event but, instead, would result from the fact that both PPI and PPRI were essentially measures of the same gated motor response. This possibility of response feedback influencing stimulus estimates was suggested previously by Blumenthal et al. (1996). However, in the present study, there is some evidence for a dissociation between startle response magnitude and estimated stimulus intensity. First, despite the near-identical startle magnitudes elicited by each stimulus in Noise-Noise or PuffPuff pairs, the second stimulus of each pair was consistently rated as being more intense than the first. Second, with acoustic stimuli, maximal PPRI was observed with $16-\mathrm{dB}$ prepulses that produced approximately $70 \%$ inhibition (PPI) of the motor response. In contrast, with tactile stimuli, no PPRI was observed with similar 16-dB prepulses, despite the fact that they produced even greater inhibition $(70 \%)$ of the motor response. Thus, the comparison of PPI versus PPRI, using intramodal and crossmodal stimuli, provided by the present study indicates that, under some conditions, PPRI cannot be fully predicted by the amount of PPI or, therefore, by the magnitude of the motor response.

Certainly, one challenge is to understand the contribution of methodological differences to differences in findings across studies. Several methodological differences clearly exist between the present study and the previous reports examining the relationship between PPI and perceived stimulus intensity. One standard approach to stimulus design in previous reports has been to utilize a range of startle stimulus intensities and a single prepulse intensity (Blumenthal et al., 1996); the opposite strategy was employed in the present study, in order to examine potential graded effects of prestimuli on PPI and PPRI. The methods for calculating PPRI and the paired-trial session design that made these methods possible differ from the methodologies in previous reports. The amount of variability in the present measures of PPRI demonstrates that more sensitive methodologies for assessing this phenomenon are needed. Nevertheless, the fact that orderly relationships between PPI and PPRI were observed in the present study indicates that PPRI may be a robust and, perhaps, even physiologically meaningful component of information processing. 
Several caveats should be considered in the interpretation of the present experiment. First, the subject population was not characterized via structured clinical interviews. Thus, it is conceivable that some subjects may have carried diagnoses associated with abnormal patterns of PPI. Toxicology screens were assessed only in a subset of the subjects. Although results did not differ between subjects with versus without negative toxicology screens, experience with our control population suggests that abnormal toxicological findings would be evident in approximately $4.5 \%$ of these unscreened subjects (Swerdlow, Geyer, Perry, Cadenhead, \& Braff, 1995). It would not be expected that a clinically mixed population would result in an artificial relationship between PPI and PPRI, although it might be predicted to increase the range of PPI and, thus, enhance the ability to detect correlations between PPI and other measures. A second possible caveat with these data is that the startle elicited by air puffs may result, in part, from the acoustic stimulus that accompanies air puff delivery (Flaten \& Blumenthal, 1998). In this case, PPRI observed in cross-modal startle trials might actually result, at least in part, from a form of loudness assimilation, as was suggested by Blumenthal et al. (1996). However, evidence against such an acoustic source of airpuff-induced startle is the substantial difference in the characteristics of PPI elicited using air puffs versus acoustic stimuli. Furthermore, with headphones in place and background white noise active, air puffs are not audible at levels of adequate intensity to produce a startle response.

The presence of the headphone and of masking noise also prevented another potential problem, that of the facilitation of a startle response to a stimulus in one modality by the simultaneous presentation of a weak stimulus in another modality (in this case, the impact of a weak sound generated by the airpuff on the blink response to an airpuff). This facilitation has been shown for various crossmodal stimulus configurations (Boelhouwer, Teurlings, \& Brunia, 1991; Sarno, Blumenthal, \& Boelhouwer, 1997), but reducing the intensity of the sound decreases the extent of the facilitation (Schmolesky, Boelhouwer, \& Blumenthal, 1996). In the present study, the headphones effectively diminished the experienced intensity of the airpuffgenerated sound to an intensity below that of the $70-\mathrm{dB}$ masking noise and, thus, prevented cross-modal reflex facilitation.

A third caveat is that comparisons of prepulse effects on acoustic versus tactile startle must consider the functional intensity of the startle stimuli. Thus, it is conceivable that prepulse effects on either perceived stimulus intensity or startle magnitude differ, depending on the intensity of the stimulus relative to the dynamic range for that sensory system. Because only a single Noise stimulus and a single Puff stimulus were used in the present study, it is not possible to generalize the observed patterns of reflex and perceptual inhibition to the full range of acoustic or tactile sensory processing. Some evidence suggests that the stimulus parameters used in the present study for Noise and Puff stimuli may be somewhat comparable, in terms of their intensities relative to the range of their respective sensory system. First, startle reflex magnitude elicited by Noise and Puff stimuli were quite similar [reflex magnitudes: mean, Noise $=84.24(S E M=4.20)$; Puff $=83.04(S E M=6.78)]$. Thus, to the extent that reflex magnitude reflects relative stimulus intensity, Noise and Puff stimuli were matched closely; in fact, this is precisely why the present stimulus parameters were selected. Second, in previous studies in our laboratory, lower levels of either acoustic (e.g., $105 \mathrm{~dB}[\mathrm{~A}]$ ) or tactile stimulus intensities (e.g., $30 \mathrm{psi}$ ) resulted in lower startle magnitudes, suggesting that intensity levels for the present Noise and Puff stimuli are neither minimal values for their respective systems nor extreme values (beyond the peak of an invertedU curve). Ultimately, however, the present findings must be interpreted with caution, since they relate to comparisons of prepulse effects across startle stimulus modalities.

Startling stimuli utilized in the present study were extremely intense, and a more sensitive assessment of prepulse effects on perceived stimulus intensity might be achieved with less intense startling (or nonstartling) stimuli. At least some effects noted in these studies, however, may not be unique to conditions of intense startling stimuli. For example, Postman (1946) noted that time errors (corresponding to the findings reported in Figure 2 of the present paper) were independent of stimulus intensity with stimuli of 35,55 , or $75 \mathrm{~dB}$. Furthermore, prepulseinduced reduction of perceived intensities in studies by three other groups have been observed in the context of either acoustic or tactile startling stimuli that are less intense than those used in the present study (Cohen et al., 1981; Peak 1939; Perlstein et al., 1993). Thus, it seems reasonable to speculate that some or all of the phenomena observed in the present study might reflect mechanisms that impact information processing of less intense phasic stimuli.

PPI is studied under very controlled and artificial conditions in the laboratory; under natural circumstances, sensorimotor gating is conceptualized as being continuously active in the waking human nervous system, contributing to our ability to segregate a continuous stream of sensory and cognitive information and to selectively allocate attentional resources to salient stimuli. The specific characteristics of an individual's gating processes are viewed to be plastic, shaped by genetic and developmental forces, but sensitive to changes in the environment, stressors, or the neurochemical and hormonal milieu of the nervous system (cf. Swerdlow, 1996). A more substantial, pathological breakdown in the gating window would be expected to impair the orderly, hierarchical processing of sensory events and to result in a flooding of sensory information, with a fragmenting impact on cognition (Braff \& Geyer, 1990; McGhie \& Chapman, 1961).

Although the present experiment identified a modest correspondence between the effects of prestimuli on reflex magnitude and perceived startle stimulus intensity, it is possible that these prepulse effects might be dissociated under other experimental conditions. In fact, Postman (1946) identified positive time errors (i.e., inhibition of 
perceived intensity of the second stimulus) with interstimulus intervals of $2 \mathrm{sec}$, which exceed by approximately $1,500 \mathrm{msec}$ the intervals that reliably produce prepulse inhibition of the startle reflex. Similarly, Cohen et al. (1981) observed reduced perception of stimulus intensity at prepulse intervals that do not result in reflex inhibition. The dissociability of prestimulus effects on reflex magnitude and perceived stimulus intensity is also evident in the nonlinear relationship between prepulse intensity and intensity ratings, despite the linear relationship between prepulse intensity and reflex magnitude, in the study of Peak (1939). Collectively, these results do not suggest that PPI per se can be interpreted as a reliable measure of sensory gating. Nonetheless, it is possible that comparisons of PPRI between clinical and control groups may extend our understanding of the relationship between PPI deficits, informationprotective brain mechanisms, and information-processing deficits in patients with neuropsychiatric disorders.

\section{REFERENCES}

Bickford-Wimer, P. C., Nagamoto, H., Johnson, R., Adler, L. E., Egan, M., Rose, G. M., \& Freedman, R. (1990). Auditory sensory gating in hippocampal neurons: A model system in the rat. Biological Psychiatry, 27, 183-192.

Blumenthal, T. D. (1997). Prepulse inhibition decreases as startle reactivity habituates. Psychophysiology, 34, 446-450.

Blumenthal, T. D., Schicatano, E. J., Chapman, J. G., Norris, C. M., \& Ergenzinger, E. R., JR. (1996). Prepulse effects on magnitude estimation of startle-eliciting stimuli and startle responses. Perception \& Psychophysics, 58, 73-80.

Boelhouwer, A. J. W., Teurlings, R. J. M. A., \& Brunia, C. H. M. (1991). The effect of an acoustic warning stimulus upon the electrically elicited blink reflex in humans. Psychophysiology, 28, 133-139.

BrafF, D. L., \& Geyer, M. A. (1990). Sensorimotor gating and schizophrenia. Archives of General Psychiatry, 47, 181-188.

BrafF, D. L., Grillon, C., \& Geyer, M. A. (1992). Gating and habituation of the startle reflex in schizophrenic patients. Archives of General Psychiatry, 49, 206-215.

Braff, D. L., Stone, C., Callaway, E., Geyer, M. A., Glick, I., \& BaLI, L. (1978). Prestimulus effects on human startle reflex in normals and schizophrenics. Psychophysiology, 15, 339-343.

Caine, S. B., Geyer, M. A., \& Swerdlow, N. R. (1992). Hippocampal modulation of acoustic startle and prepulse inhibition in rats. Pharmacology, Biochemistry \& Behavior, 43, 1201-1208.

Castellanos, F. X., Fine, E. J., Kaysen, D. L., Kozuch, P. L., Hamburger, S. D., Rapoport, J. L., \& Hallett, M. (1996). Sensorimotor gating in boys with Tourette's syndrome and ADHD: Preliminary results. Biological Psychiatry, 39, 33-41.

Cohen, M. E., Hoffman, H. S., \& StitT, C. L. (1981). Sensory magnitude estimation in the context of reflex modification. Journal of Experimental Psychology: Human Perception \& Performance, 7, $1363-$ 1370.

Conrad, A. J., Abebe, T., Austin, R., Forsythe, S., \& Scheibel, A. B. (1991). Hippocampal pyramidal cell disarray in schizophrenia as a bilateral phenomenon. Archives of General Psychiatry, 48, 413-417.
Flaten, M. A., \& Blumenthal, T. D. (1998). A parametric study of the separate contributions of the tactile and acoustic components of airpuffs to the blink reflex. Biological Psychology, 48, 227-234.

Graham, F. (1975). The more or less startling effects of weak prestimuli. Psychophysiology, 12, 238-248.

Ison, J. R., MCAdam, D. W., \& Hammond, G. R. (1973). Latency and amplitude changes in the acoustic startle reflex produced by variations in auditory prestimulation. Physiology \& Behavior, 10, 1035-1039.

MCGhiE, A., \& Chapman, J. (1961). Disorders of attention and perception in early schizophrenia. British Journal of Medical Psychology, $34,102-116$.

Norris, C. M., \& Blumenthal, T. D. (1996). A relationship between inhibition of the acoustic startle response and the protection of prepulse processing. Psychobiology, 24, 160-168.

PEAK, H. (1939). Time order error in successive judgements and in reflexes: I. Inhibition of the judgement and the reflex. Journal of Experimental Psychology, 25, 535-565.

Perlstein, W. M., Fiorito, E., Simons, R. F., \& Graham, F. K. (1993). Lead stimulation effects on reflex blink, exogenous brain potentials, and loudness judgments. Psychophysiology, 30, 347-358.

Postman, L. (1946) The time-error in auditory perception. American Journal of Psychology, 59, 193-219.

Sarno, A. J., Blumenthal, T. D., \& Boelhouwer, A. J. W. (1997). Modification of the electrically elicited eyeblink by acoustic, visual, and vibrotactile pulses. Psvchobiology, 25, 253-265.

SChmolesky, M. T., Boelhouwer, A. J. W., \& Blumenthal, T. D. (1996). The effect of acoustic pulse intensity upon the electrically elicited blink reflex at positive and negative stimulus onset asynchronies. Biological Psychology, 44, 69-84.

Siegel, C., Waldo, M., Mizner, G., Adler, L. E., \& Freedman, R. (1984). Deficits in sensory gating in schizophrenic patients and their relatives: Evidence obtained with auditory evoked responses. Archives of General Psychiatry, 41, 607-612.

SWERDLOW, N. R. (1996). Cortico-striatal substrates of cognitive, motor and sensory gating: Speculations and implications for psychological function and dysfunction. In J. Panksepp (Ed.), Advances in biological psychiatry (Vol. 2, pp. 179-208). Greenwich, CT: JAI Press.

Swerdlow, N. R., Filion, D., Geyer, M. A., \& BrafF, D. L. (1995). "Normal" personality correlates of sensorimotor, cognitive and visuospatial gating. Biological Psychiatry, 37, 286-299.

Swerdlow, N. R., Geyer, M. A., Perry, W., Cadenhead, K., \& BrafF, D. L. (1995). Drug screening in "normal" controls. Biological Psychiatry, 38, 123-124.

Swerdlow, N. R., Hartman, P. L., \& Auerbach, P. P. (1997). Changes in sensorimotor inhibition across the menstrual cycle: Implications for neuropsychiatric disorders. Biological Psychiatry, 41, 452-460.

SWERdLOW, N. R., LiPSKa, B. K., WeInBerger, D. R., BrafF, D. L., JASKIW, G. E., \& GEYER, M. A. (1995). Increased sensitivity to the gating-disruptive effects of apomorphine after lesions of the medial prefrontal cortex or ventral hippocampus in adult rats. Psychopharmacology, 122, 27-34.

Swerdlow, N. R., Paulsen, J., Braff, D. L., Butters, N., Geyer, M. A., \& SWENSON, M. R. (1995). Impaired prepulse inhibition of acoustic and tactile startle in patients with Huntington's disease. Journal of Neurology, Neurosurgery \& Psychiatry, 58, 192-200.

WOODWORTH, R. S., \& SCHLOSBERG, H. (1954). Experimental psychology. New York: Holt, Rinehart \& Winston.

(Manuscript received December 16, 1998; revision accepted for publication March 29, 1999.) 